\title{
A SPHERE THEOREM FOR REVERSE VOLUME PINCHING ON EVEN-DIMENSIONAL MANIFOLDS
}

\author{
LESLIE COGHLAN AND YOE ITOKAWA
}

(Communicated by Jonathan M. Rosenberg)

\begin{abstract}
Let $M$ be a compact simply connected riemannian manifold of even dimension $d$. It is well known that if the sectional curvature of $M$ lies in the range $(0, \lambda]$, then $M$ has volume greater than or equal to that of the $d$-dimensional euclidean sphere $S_{\lambda}^{d}$ of constant curvature $\lambda$. We prove that if the volume of $M$ is no greater than $3 / 2$ times that of $S_{\lambda}^{d}$, then $M$ is homeomorphic with the sphere.
\end{abstract}

\section{INTRODUCTION}

Of crucial importance in the proof of the classical sphere theorem of H. E. Rauch, M. Berger, and W. Klingenberg (see [4, Chapter 6]) is the injectivity radius estimate of Klingenberg [13] and J. Cheeger and D. Gromoll [5] (cf. also [14]).

(1.0) Lemma. Let $M$ be a compact simply connected riemannian manifold $M$ with sectional curvature $K$. If either the dimension $d$ of $M$ is odd and $K$ lies in the range $\lambda \geq K \geq \lambda / 4>0$, or if $M$ is even and $\lambda \geq K>0$ for some constant $\lambda$, then the injectivity radius $i(M)$ is bounded from below by $\pi / \sqrt{\lambda}$.

Note that the assumption on the upper bound for the sectional curvature is a restrictive condition for odd dimensions but merely a normalization for even dimensions. In spite of this strong structural restriction, our knowledge at present on even-dimensional positively curved manifolds still seems rather meager.

Let us denote by $\sigma(d)$ the volume of the $d$-dimensional unit euclidean sphere $S_{1}^{d}$ in $\mathbb{R}^{d+1}$. In this paper, we prove

(1.1) Main Theorem. Let $M$ be a compact simply connected riemannian manifold of even dimension $d$. Suppose that $M$ has positive sectional curvature with $\operatorname{Max} K \leq \lambda, \lambda$ constant, and that its volume $V(M)$ is no larger than

Then $M$ is homeomorphic to $S_{1}^{d}$.

$$
3 / 2 \cdot \sigma(d) / \lambda^{d / 2} \text {. }
$$

Received by the editors October 27, 1989.

1980 Mathematics Subject Classification (1985 Revision). Primary 53C20.

This work was supported in part by NSF EPSCoR grant RII-8610669. 
We note that (1.0) combined with the well-known volume comparison theorem of R. Bishop [2, $\S 11.10]$ and P. Günther [10], easily gives

Theorem. Let $M$ be a compact d-dimensional connected riemannian manifold of positive sectional curvature $K, \lambda \geq K \geq \kappa$ for some constants $\lambda, \kappa$. Then $V(M) \leq \sigma(d) / \kappa^{d / 2}$, and, if in addition $d$ is even-dimensional and simply connected, then also $V(M) \geq \sigma(d) / \lambda^{d / 2}$. Moreover, if equality holds in either of the above, then $M$ is isometric to the d-dimensional sphere of constant curvature.

In this context, (1.1) forms a counterpart in even dimensions to the following volume pinching theorem of C. Heim [11], K. Grove and K. Shiohama [9] (cf. also $[1,15])$.

Theorem. Let $M$ be a compact connected riemannian manifold of curvature $K \geq \kappa>0$ and dimension $d$ such that $V(M)>\sigma(d) / 2 \kappa^{d / 2}$. Then $M$ is homeomorphic to $S_{1}^{d}$.

For more recent results in the structure of manifolds with volume restricted from below, see Shiohama [18] (cf. also [12]) and Y. Otsu, K. Shiohama, and T. Yamaguchi [16].

We also point out

(1.2) Theorem. In each even dimension $d$, given any real $\Psi>0$, there exist only finitely many different diffeomorphism types of compact connected manifolds that admit riemannian metrics of positive sectional curvature satisfying $V(M) \leq$ $\Psi /[\operatorname{Max} K]^{d / 2}$.

This is because in both the original proof by Cheeger [3] and in the more recent proof by S. Peters [17] of the classical finiteness theorem (cf. [4, 7.37]), the assumption on diameter is only used to obtain an upper volume estimate (which is in turn used to obtain an estimate on the number of balls in a convex covering of such a manifold) and to obtain an injectivity radius estimate. In our case, both of these are already given. In fact, once an estimate is given for the number of balls of fixed radius that are needed to cover $M$, the diameter can be easily estimated from above. See, also, a more recent work of K. Grove, P. Petersen V, and J.-Y. Wu [8].

When combined with the eigenvalue comparison theorem of S. Y. Cheng [6], this yields the following interesting corollary which (surprisingly!) seems to have gone previously unnoticed.

(1.3) Corollary. In each even dimension $d$, given any positive integer $n$ and a real $\Lambda>0$, there exist only finitely many diffeomorphism types for compact manifolds which admit metrics of positive curvature such that $\lambda_{n}(M)$, nth nonzero eigenvalue for the Laplacian on functions, satisfies $\lambda_{n}(M) \geq \Lambda(\operatorname{Max} K)$.

Throughout this paper, we follow [4] as the reference for all definitions and notations. We split the proof into two steps. First, we prove the theorem for 
the case of strict inequality,

$$
V(M)<\frac{3}{2} \cdot \sigma(d) / \lambda^{d / 2},
$$

for which the argument is considerably simpler. We will then give an argument for the case of equality. Even though this represents only a marginal improvement in the theorem quantitatively, it does serve as evidence to suggest the existence of a $\delta>\frac{3}{2}$ such that the inequality $V(M)<\delta \cdot \sigma(d) / \lambda^{d / 2}$ still implies homeomorphism with the sphere. The complex projective plane, when properly normalized, is seen to have the volume $3 \sigma(4)$. This leads us to suspect that $\delta=3$ is the sharp constant.

\section{Proof. The Strict INEQUality CASE}

Let $M$ satisfy the assumptions described in (1.1). In this section, assume that $V(M)>\sigma(d) / \lambda^{d / 2}$. By scaling the metric, if necessary, we may also assume that $\lambda=1$. Choose the points $p$ and $q$ on $M$ so that $\rho(p, q)=$ the diameter of $M$. We first recall, from Bishop and Günther (loc. cit.), the following

$$
\text { Lemma. For any } p \in M, V\left(B_{\pi / 2}(p)\right) \geq \sigma(d) / 2 \text {. }
$$

We then have

(2.1) Lemma. There exists some $\varepsilon>0$ such that the two closed balls $B_{\pi-\varepsilon}(p)^{-}$ and $B_{\pi-\varepsilon}(q)^{-}$cover $M$.

Proof. By the injectivity radius estimate (1.0) and our choice for $p$ and $q$, $\rho(p, q) \geq \pi$. Therefore, $B_{\pi / 2}(p)$ and $B_{\pi / 2}(q)$ are mutually disjoint. Hence, by $(2.0)$,

$$
\begin{aligned}
V\left(M \backslash\left[B_{\pi / 2}(p) \cup B_{\pi / 2}(q)\right]\right) & =V(M)-V\left(B_{\pi / 2}(p)\right)-V\left(B_{\pi / 2}(q)\right) \\
& <\frac{3}{2} \sigma(d)-\frac{1}{2} \sigma(d)-\frac{1}{2} \sigma(d)=\frac{1}{2} \sigma(d) .
\end{aligned}
$$

Now, if $B_{\pi / 2}(p)^{-} \cup B_{\pi / 2}(q)^{-}=M$, there is nothing to prove. Assume that $x \in$ $M \backslash\left[B_{\pi / 2}(p)^{-} \cup B_{\pi / 2}(q)^{-}\right]$. Again, by $(2.0), V\left(B_{\pi / 2}(x)\right) \geq \sigma(d) / 2$. This means that $B_{\pi / 2}(x)$ cannot be contained wholly in the set $M \backslash\left[B_{\pi / 2}(p) \cup B_{\pi / 2}(q)\right]$. By the open condition, there exists $\varepsilon>0$ such that $B_{\pi / 2-\varepsilon}(x)$ is still not contained in the set $M \backslash\left[B_{\pi / 2}(p) \cup B_{\pi / 2}(q)\right]$. In fact, $\varepsilon$ can be chosen so as not to depend on the choice of $x$ in $M \backslash\left[B_{\pi / 2}(p)^{-} \cup B_{\pi / 2}(q)^{-}\right]$. For each such $x$, let

$$
\varepsilon(x):=\frac{1}{2} \sup \left\{\delta: B_{\pi / 2-\delta}(x) \not \subset M \backslash\left(B_{\pi / 2}(p) \cup B_{\pi / 2}(q)\right)\right\} .
$$

Then $\varepsilon(x)$ depends continuously on $x$ and tends to $\pi / 4$ as $x$ approaches the boundary of the set $M \backslash\left[B_{\pi / 2}(p)^{-} \cup B_{\pi / 2}(q)^{-}\right]$. Hence, $\varepsilon:=\inf _{x} \varepsilon(x)>0$.

So, there is an $\varepsilon$ depending only on $M$ such that $B_{\pi / 2-\varepsilon}(x)^{-}$must intersect either $B_{\pi / 2}(p)^{-}$or $B_{\pi / 2}(q)^{-}$. By the triangle inequality, this implies that either $\rho(x, p) \leq \pi-\varepsilon$ or $\rho(x, q) \leq \pi-\varepsilon$.

Cf. [12], where the second-named author used a similar argument. 
By (1.0), $i(M) \geq \pi$, so each of the balls $B_{\pi-\varepsilon}(p)^{-}$and $B_{\pi-\varepsilon}(q)^{-}$is diffeomorphic to a closed $d$-dimensional disk and every geodesic emanating from $p$ reaches $B_{\pi}(p)$ as a length minimizing curve. It is now a standard argument to establish an explicit homeomorphism from $S^{d}$ onto $M$. For example, the proof of 6.4 in [4] follows through as it stands. Alternatively, one can appeal to a nontrivial theorem of J. Stallings [19].

\section{Proof. The equality case}

We continue with the assumptions described in (1.1). We now suppose that $V(M)=\frac{3}{2} \sigma(d)$. Note that if we can establish Lemma (2.1), the rest of the argument follows. So assume that Lemma (2.1) is false. First we establish a purely topological lemma, as follows:

(3.1) Lemma. Let $R$ be a connected topological space and let $A, B$, and $C$ be mutually disjoint open subsets of $R$ such that their boundaries $\partial A, \partial B$, and $\partial C$ are each nonempty and connected in $R$, and the union of each one with the closures of the other two cover $R$; i.e., $A \cup B^{-} \cup C^{-}=A^{-} \cup B \cup C^{-}=$ $A^{-} \cup B^{-} \cup C=R$. Then $\partial A \cap \partial B \cap \partial C \neq \varnothing$.

Proof. From the first and last assumptions, it follows that each of the three sets $\partial A, \partial B$, and $\partial C$ is contained in the union of the other two boundary sets. First we contend that neither of the two, $\partial A \cap \partial C$ nor $\partial B \cap \partial C$, is empty. In fact, if $\partial A \cap \partial C=\varnothing$, we then must have $\partial A \subset \partial B$ and $\partial C \subset \partial B$. Therefore, $\partial A \cap \partial B \neq \varnothing, \partial C \cap \partial B \neq \varnothing$, and $(\partial A \cap \partial B) \cup(\partial C \cap \partial B)=\partial B$. On the other hand, $\partial A \cap \partial B$ and $\partial C \cap \partial B$ are both closed in $\partial B$, so this contradicts the connectedness of $\partial B$. Now reverse the roles of $A$ and $B$ to establish the claim.

Now, assume that $\partial A \cap \partial B \cap \partial C=(\partial A \cap \partial C) \cap(\partial B \cap \partial C)=\varnothing . \quad \partial C \subset$ $\partial A \cup \partial B$ implies $\partial C \subset(\partial A \cup \partial B) \cap \partial C=(\partial A \cap \partial C) \cup(\partial B \cap \partial C)$, so that $\partial C=(\partial A \cap \partial C) \cup(\partial B \cap \partial C)$. This time, the closedness of $\partial A \cap \partial C$ and $\partial B \cap \partial C$ contradicts the connectedness of $\partial C$.

Now, choose $p, q \in M$ as in the previous section. If the statement of Lemma (2.1) under the weaker hypothesis is false, then there must exist a point $x \in M \backslash\left[B_{\pi / 2}(p)^{-} \cup B_{\pi / 2}(q)^{-}\right]$such that the three balls $B_{\pi / 2}(p), B_{\pi / 2}(q)$, and $B_{\pi / 2}(x)$ are pairwise disjoint. On the other hand, by the volume comparison $(2.0)$, we must still have

$$
B_{\pi / 2}(p)^{-} \cup B_{\pi / 2}(q)^{-} \cup B_{\pi / 2}(x)^{-}=M .
$$

Moreover, by (1.0), $i(M) \geq \pi$, so the three boundary sets $\partial B_{\pi / 2}(p), \partial B_{\pi / 2}(q)$, and $\partial B_{\pi / 2}(x)$ are each diffeomorphic to $S^{d-1}$ and hence connected. We may therefore apply Lemma (3.1) to obtain a point $r$ in the triple intersection $\partial B_{\pi / 2}(p) \cap \partial B_{\pi / 2}(q) \cap \partial B_{\pi / 2}(x)$. Let $\gamma$ and $\eta$ be the unique minimal geodesics joining $p$ to $r$ and $q$ to $r$, respectively. We assume that each curve 
is parametrized by arclength. Since $B_{\pi / 2}(x)^{-} \subset M \backslash B_{\pi / 2}(p)$, by the first variation of distance from $p$ to the smooth hypersurface $\partial B_{\pi / 2}(x)$, we see that $\gamma$ is perpendicular to $\partial B_{\pi / 2}(x)$ at $r$. Likewise, $\eta$ is also perpendicular to $\partial B_{\pi / 2}(x)$ at $r$. By the uniqueness of the geodesics, $\gamma \equiv \eta$ and $p=q$. This contradicts our choice of $p$ and $q$. Therefore, the conclusion of Lemma (2.1) must hold under the weaker assumption on the volume of $M$, and $M$ is still covered by two topological disks.

\section{REFERENCES}

1. M. Berger, On the diameter of some riemannian manifolds, Technical Report, University of California, Berkeley, 1962.

2. R. L. Bishop and R. J. Crittenden, Geometry of manifolds. Academic Press, New York and London, 1964.

3. J. Cheeger, Finiteness theorems for riemannian manifolds, Amer. J. Math. 92 (1970), 61-74.

4. J. Cheeger and D. G. Ebin, Comparison theorems in Riemannian geometry, North-Holland, Amsterdam, 1975.

5. J. Cheeger and D. Gromoll, On the injectivity radius of $\frac{1}{4}$-pinched manifolds, J. Differential Geom. 15 (1980), 437-442.

6. S. Y Cheng, Eigenvalue comparison theorems and its geometric applications, Math. Z. 143 (1975), 289-297.

7. K. Grove and P. Petersen V, Bounding homotopy types by geometry, Ann. of Math. 128 (1988), 195-206.

8. K. Grove, P. Petersen V, and J.-Y. Wu, Geometric finiteness theorem via controlled topology, Inv. Math. 99 (1990), 205-213.

9. K. Grove and K. Shiohama, A generalized sphere theorem, Ann. of Math. 106 (1977), 201211.

10. P. Günther, Einige Sätze über das Volumenelement eines Riemannschen Raumes, Publ. Math. Debrecen 7 (1960), 78-93.

11. C. Heim, Une borne pour la longeur des géodésiques periodiques d'une variété riemannienne compacte, Thesis, Université Paris, 1971.

12. Y. Itokawa, The topology of certain riemannian manifolds with positive Ricci curvature, J. Differential Geom. 18 (1983), 151-155.

13. W. Klingenberg, Contributions to riemannian geometry in the large, Ann. of Math. 69 (1950), 654-666.

14. W. Klingenberg and T. Sakai, Injectivity radius estimate for $\frac{1}{4}$-pinched manifolds, Arch. Math. 34 (1980), 371-376.

15. T. Nagayoshi and Y. Tsukamoto, On positively curved riemannian manifolds with bounded volume, Tôhoku Math. J. 25 (1973), 213-218.

16. Y. Otsu, K. Shiohama, and T. Yamaguchi, A new version of differential sphere theorem, Inv. Math. 98 (1989), 219-228.

17. S. Peters, Cheeger's finiteness theorem for diffeomorphism classes of riemannian manifolds, J. Reine Angew. Math. 394 (1984), 77-82.

18. K. Shiohama, A sphere theorem for manifolds of positive Ricci curvature, Trans. Amer. Math. Soc. 275 (1983), 811-819.

19. J. Stallings, Polyhedral homotopy spheres, Bull. Amer. Math. Soc. 66 (1960), 485-488.

Department of Mathematics, School of Natural Sciences and Mathematics, UniverSity of Alabama, Birmingham, Alabama 35294 